\title{
Transient large heat advection in fractured rock: a zero-thickness interface formulation
}

\author{
A.Pérez, I. Carol, P. Prat \\ Div. of Geotechnical Engineering and Geo-Sciences \\ ETSECCPB (School of Civil Engineering), \\ UPC (Universitat Politècnica de Catalunya) \\ 08034 Barcelona \\ e-mail: adria.perez@upc.edu, ignacio.carol@upc.edu,pere.prat@upc.edu
}

\begin{abstract}
Two of the main mechanisms of heat transport in engineering problems are diffusion and advection. In a FEM context it is well known that if advection dominates over diffusion (Péclet number $\mathrm{Pe}>1$ ), traditional Galerkin formulations may lead to oscillatory results [1], although in many practical engineering situations, such as the case of geological materials, fluid velocities generally remain small due to the low permeability of the pore system and this problem may be ignored [2].

However, in the presence of preference paths of fluid circulation this situation may change. The existence of open fractures or cracks may produce high fluid velocities through them in comparison with those found in the surrounding porous medium. This situation can lead to exceeding the limit condition $P e>1$. In a FEM context, these preferential paths or cracks are sometimes modelled by using double-nodded zero-thickness interface elements. Thus, the paper discusses these concepts and presents a zero-thickness interface FE formulation for large advection, which consists of an implicit extension of Taylor's explicit formulation for the transient problem based on characteristics [1], which has been developed for traditional continuum elements in its explicit form. Some verification examples are also presented, showing that even in the extreme case of pure advection $(P e \rightarrow \infty)$, the formulation exhibits a good performance to represent the heat transport by the fluid along the mesh.
\end{abstract}

\section{REFERENCES}

[1] Zienkiewicz, O. \& Taylor, R., The Finite Element Method: Vol 3. Fluid Dynamics. Fifth ed. Oxford: Butterworth-Heinemann (2000).

[2] Huysmans, M. \& Dassargues, A. "Review of the use of Péclet numbers to determine the relative importance of advection and diffusion in low permeability environments". Hydrogeology Journal. Vol. 13. Num 5-6, pp. 895-904. Springer-Verlag (2004). 